\title{
Multivariate Tensor-Based Brain Anatomical Surface Morphometry via Holomorphic One-Forms
}

\author{
Yalin Wang ${ }^{1,2}$, Tony F. Chan ${ }^{2}$, Arthur W. Toga ${ }^{1}$, and Paul M. Thompson ${ }^{1}$ \\ 1 Lab. of Neuro Imaging, UCLA School of Medicine, Los Angeles, CA 90095, USA \\ 2 Mathematics Department, UCLA, Los Angeles, CA 90095, USA \\ ylwang@loni.ucla.edu
}

\begin{abstract}
Here we introduce multivariate tensor-based surface morphometry using holomorphic one-forms to study brain anatomy. We computed new statistics from the Riemannian metric tensors that retain the full information in the deformation tensor fields. We introduce two different holomorphic one-forms that induce different surface conformal parameterizations. We applied this framework to 3D MRI data to analyze hippocampal surface morphometry in Alzheimer's Disease (AD; 26 subjects), lateral ventricular surface morphometry in HIV/AIDS (19 subjects) and cortical surface morphometry in Williams Syndrome (WS; 80 subjects). Experimental results demonstrated that our method powerfully detected brain surface abnormalities. Multivariate statistics on the local tensors outperformed other TBM methods including analysis of the Jacobian determinant, the largest eigenvalue, or the pair of eigenvalues, of the surface Jacobian matrix.
\end{abstract}

\section{Introduction}

Surface-based methods have been extensively used to study structural features of the brain, such as cortical gray matter thickness, complexity, and deformation over time 1]. Also, deformation-based morphometry (DBM) 2] directly uses 2D or $3 \mathrm{D}$ deformations obtained from the nonlinear registration of brain images to infer local differences in brain volume or shape. Tensor-based morphometry (TBM) 3] tends to examine spatial derivatives of the deformation maps registering brains to a common template, constructing morphological tensor maps such as the Jacobian determinant, torsion or vorticity. DBM, by contrast, tends to analyze 3D displacement vector fields encoding relative positional differences across subjects. One advantage of TBM for surface morphometry is that surfaces are commonly parameterized using grids from which local deformation tensors can be naturally derived - TBM can even make use of the Riemannian surface metric to characterize local anatomical changes.

In computational differential geometry, a holomorphic one-form [4] can be represented as a pair of scalars on each edge of a discrete mesh structure. The holomorphic one-form is an intrinsic, coordinate-free formulation. It provides a practical way to induce conformal parameterizations on surfaces and compute 
surface-to-surface registrations. The holomorphic one-forms are computed by solving linear systems so the computation is very stable.

In this paper, we present a multivariate TBM framework and apply it to detect abnormal areas on anatomical structures in the brain represented as surfaces, parameterized using differential forms (holomorphic one-forms). We performed three empirical studies of brain abnormalities in Williams syndrome (WS), Alzheimer's Disease (AD), and HIV/AIDS. We studied hippocampal surface deformation associated with $\mathrm{AD}$, and lateral ventricular surface deformation associated with HIV/AIDS. The proposed multivariate TBM detected areas of statistically significant deformation even in relatively small test datasets - one compares 12 subjects with $\mathrm{AD}$ to a group of 14 matched healthy controls and the other compares 11 subjects with HIV/AIDS to 8 matched healthy controls. We also detected regions with statistically significant abnormal surface morphology in cortical data from 40 individuals with WS versus 40 matched healthy controls. For comparison, we also applied another three map-based surface statistics to the same three brain anatomical surface datasets. Our goal was to show that the proposed multivariate TBM had more detection power by detecting consistent but more statistically significant areas of abnormal brain structure. Also note that the proposed multivariate TBM framework is simple and general. The Jacobian matrix can be easily computed by Equation 11. Potentially it can take results from any surface registration methods for further morphometry study.

\section{Methods}

Holomorphic one-forms, a structure used in differential geometry, can be used to generate both canonical conformal parametrization [5] and slit conformal parameterization [6] on 3D anatomical surfaces. The obtained parameterization maximizes the uniformity of the induced grid over the entire domain (see [5]6] for a more detailed algorithm description).

Suppose $\phi: S_{1} \rightarrow S_{2}$ is a map from surface $S_{1}$ to surface $S_{2}$. The derivative map of $\phi$ is the linear map between the tangent spaces, $d \phi: T M(p) \rightarrow T M(\phi(p))$. In practice, smooth surfaces are usually approximated by triangle meshes. The derivative map $d \phi$ is approximated by the linear map from one face $\left[v_{1}, v_{2}, v_{3}\right]$ to another one $\left[w_{1}, w_{2}, w_{3}\right]$. First, we isometrically embed the triangle $\left[v_{1}, v_{2}, v_{3}\right]$, $\left[w_{1}, w_{2}, w_{3}\right]$ onto the plane $\mathbb{R}^{2}$; the planar coordinates of the vertices of $v_{i}, w_{j}$ are denoted using the same symbols $v_{i}, w_{j}$. Then we explicitly compute the linear matrix for the derivative map, $d \phi$, which is the Jacobian matrix of $\phi$,

$$
d \phi=\left[w_{3}-w_{1}, w_{2}-w_{1}\right]\left[v_{3}-v_{1}, v_{2}-v_{1}\right]^{-1} .
$$

In our work, we use multivariate statistics on deformation tensors [7] and adapt the concept to surface tensors. Let $J$ be the derivative map and define the deformation tensors as $S=\left(J^{T} J\right)^{1 / 2}$. Instead of analyzing shape change based on the eigenvalues of the deformation tensor, we consider a new family of metrics, the "Log-Euclidean metrics" 8. These metrics make computations on tensors easier to perform, as they are chosen such that the transformed values form a 
vector space, and statistical parameters can then be computed easily using the standard formulae for Euclidean spaces.

We apply Hotelling's $T^{2}$ test on sets of values in the log-Euclidean space of the deformation tensors. Given two groups of $n$-dimensional vectors $S_{i}, i=1, \ldots, p$, $T_{j}, j=1, \ldots, q$, we use the Mahalanobis distance $M$ to measure the group mean difference, $M=(\log \bar{S}-\log \bar{T}) \Sigma^{-1}(\log \bar{S}-\log \bar{T})$, where $\bar{S}$ and $\bar{T}$ are the means of the two groups and $\Sigma$ is the combined covariance matrix of the two groups.

\section{$3 \quad$ Experimental Results}

We applied the multivariate TBM method to various anatomical surfaces extracted from 3D MRI scans of the brain. For registering anatomical surfaces across subjects, we found that conformal slit mapping works well for cortical surface registration because the overall shape of a cortex is close to a sphere and the landmark curve locations are generally similar to each other. On the other hand, holomorphic flow segmentation works better for parameterizing long, cylinderlike shapes, such as hippocampal and lateral ventricular surfaces. In the light of this observation, we used a canonical holomorphic one-form to conformally map hippocampal and lateral ventricular surfaces to a set of planar rectangles (subsection 3.1 and 3.2); and we used slit map conformal parameterization to conformally map cortical surfaces to multiply connected domains (subsection 3.3). Through the parameter domain, we can register surfaces by using a constrained harmonic map [5].

In this paper, the segmentations are regarded as given, and results are from automated and manual segmentations detailed in other prior works 91011.

\subsection{Multivariate Tensor-Based Morphometry on Hippocampal Surfaces: Application to Alzheimer's Disease}

The hippocampal surface is a structure in the medial temporal lobe of the brain. Parametric shape models of the hippocampus are commonly developed for tracking shape differences or longitudinal atrophy in disease. Many prior studies, e.g., 9], have shown that there is atrophy as the disease progresses. In our method, we leave two holes on the front and back of the hippocampal surface, representing its anterior junction with the amygdala, and its posterior limit as it turns into the white matter of the fornix. It can then be logically represented as an open boundary genus-one surface, i.e., a cylinder. Its canonical holomorphic one-form can be easily computed. By integrating this holomorphic one-form, it can be conformally mapped to a rectangle and registered by using a constrained harmonic map.

Figure1(a)-(d) illustrate our experimental results on a group of hippocampal surface models extracted from 3D brain MRI scans of 12 AD individuals and 14 control subjects 9]. After surface registration, we ran a permutation test with 5000 random assignments of subjects to groups to estimate the statistical significance of the areas with group differences in surface morphometry. We also 

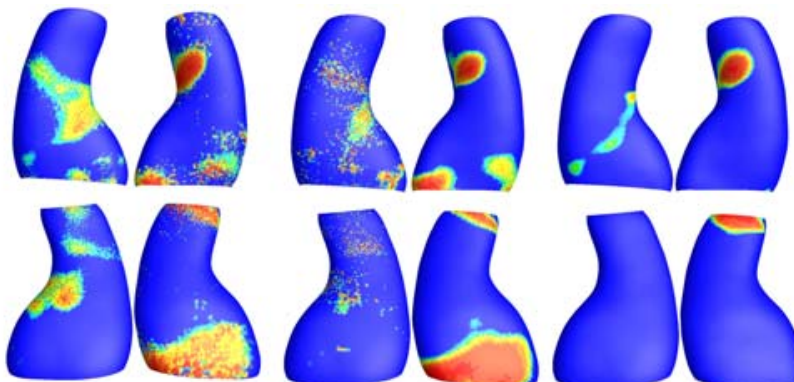

(R)

(L) (a) Multivariate TBM
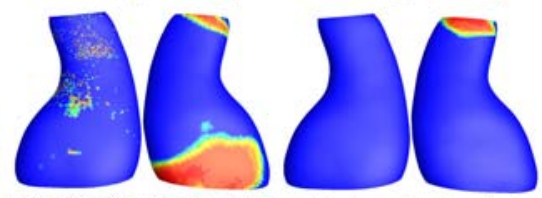

(b) Pair of eigenvalues (c) Determinant of $\mathrm{J}$

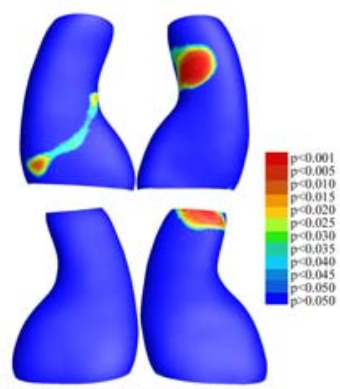

(d) Largest eigenvalue
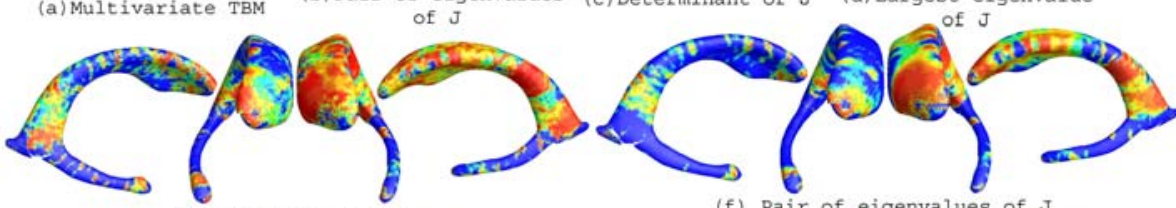

of $\mathrm{J}$

(f) Pair of eigenvalues of $\mathrm{J}$

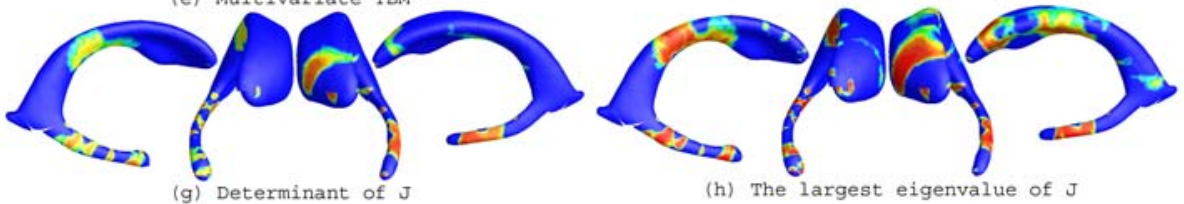

Fig. 1. (a) \& (b) illustrate canonical conformal parameterization results. (c)-(j) show statistical $p$-map results of various TBMs on (1) a group of hippocampal surfaces from $12 \mathrm{AD}$ patients and 14 matched controls((c)-(f)); (2) a group of lateral ventricular surfaces from $11 \mathrm{HIV} /$ AIDS patients and 8 matched controls $((\mathrm{g})-(\mathrm{j}))$. On the color-coded scale, non-blue colors denote the vertices where there is a significant statistical difference, at the $p=0.05$ level. Multivariate TBM detected anatomical differences more powerfully than other TBM statistics. Overall statistical significance values (corrected for multiple comparisons) are listed in Table 1.

used a statistical threshold of $p=0.05$ at each surface point to compute the supra-threshold surface area, and we estimate the overall significance of the experimental results by using a non-parametric permutation test to establish an empirical null distribution for this surface area [10. Although the samples sizes are small, we still detected relatively large statistically significant areas, consistent with prior findings 9]. The overall statistical significance $p$-values, based on permutation testing (and therefore corrected for multiple comparisons), were 0.0198 for the left hippocampal surface and 0.0410 for the right hippocampal surface (Figure 1 (a)).

\subsection{Multivariate Tensor-Based Morphometry of the Ventricular Surface in HIV/AIDS}

The lateral ventricles - fluid-filled structures deep in the brain - are often enlarged in disease and can provide sensitive measures of disease progression [11]. Ventricular changes reflect atrophy in surrounding structures, so ventricular measures 
and surface-based maps provide sensitive assessments of tissue reduction that correlate with cognitive deterioration in illnesses. However, the concave shape, complex branching topology and extreme narrowness of the inferior and posterior horns have made it difficult for surface parametrization approaches to impose a grid on the entire structure without introducing significant area distortion. To model the lateral ventricular surface, we automatically locate and introduce three cuts on each ventricle. The cuts are motivated by examining the topology of the lateral ventricles, in which several horns are joined together at the ventricular "atrium" or "trigone". We call this topological modeling step, interpreting the ventricles as a set of connected, simpler surfaces, a topology optimization operation. The topology optimization helps to enable a uniform parametrization in some areas that otherwise are very difficult to capture with usual parametrization methods. After the topology is modeled in this way, a lateral ventricular surface, in each hemisphere, becomes an open boundary surface with 3 boundaries. We computed the canonical holomorphic one-form 5 . With holomorphic flow segmentation [5], each lateral ventricular surface can be divided into 3 pieces. Although surface geometry is widely variable across subjects, the zero point locations are intrinsically determined by the surface conformal structures, and the partitioning of the surface into component meshes is highly consistent across subjects. The automatic surface segmentation result for a lateral ventricular surface is similar to the manual surface segmentation results used in prior research [11; even so it improves on past work as it avoids arbitrarily chopping the surface into 3 parts using a fixed coronal plane. After the surface segmentation, each lateral ventricular surface is divided to three surfaces, each topologically equivalent to a cylinder. For each piece, we again applied the holomorphic flow algorithm to it and conformally mapped it to a rectangle. Then we registered each part by a constrained harmonic map. Since all ventricle surfaces are similar and the critical graph is intrinsic to surface, the surface segmentation results are very consistent. It provides a stable surface registration scheme for lateral ventricular surfaces.

In our experiments, we compared ventricular surface models extracted from 3D brain MRI scans of $11 \mathrm{HIV} / \mathrm{AIDS}$ individuals and 8 control subjects [11. After surface registration, we computed the surface Jacobian matrix and applied multivariate tensor-based statistics to study differences in ventricular surface morphometry. We ran a permutation test with 5000 random assignments of subjects to groups to estimate the statistical significance of the areas with group differences in surface morphometry. We also used a statistical threshold of $p=0.05$ at each surface point to estimate the overall significance of the experimental results by non-parametric permutation test [10]. The experimental results are shown in Figure 1(e). Although sample sizes are small, we still detected large statistically significant areas, consistent with prior findings [1]. The overall statistical significance $p$-values, based on permutation testing, were 0.0022 for the left lateral ventricle and 0.008 for the right lateral ventricle. 


\subsection{Multivariate Tensor-Based Morphometry on Cortical Surfaces of Subjects with Williams Syndrome}

We also applied our framework to a cortical surface morphometry study of the brain. We analyzed cortical surface models extracted from 3D brain MRI scans of $40 \mathrm{WS}$ individuals and 40 healthy control subjects [10. We selected a set of 10 landmark curves: the Central Sulcus, Superior Temporal Sulcus Main Body, Inferior Frontal Sulcus, Middle Frontal Sulcus, Inferior Temporal Sulcus, Secondary Intermediate Sulcus, Transverse Occipital Sulcus, Inferior Callosal Outline Segment, Superior Rostral Sulcus, and Subparietal Sulcus. The definitions of these anatomical lines are reported in [12. After we cut the cortical surface open along the selected landmark curves, a cortical surface became topologically equivalent to an open boundary genus-9 surface. With holomorphic one-forms, the surface can be conformally mapped to an annulus with 8 concentric arcs 6 . Based on surface conformal parameterization, we use the landmark curves as the boundary condition and perform a constrained harmonic map to register the cortical surfaces. For each point on the cortical surface, we ran a permutation test (non-parametric t test) with 5,000 random assignments of subjects to groups to estimate the statistical significance of the areas with group differences in surface morphometry. Also, given a statistical threshold of $p=0.05$ at each surface point, we applied permutation test to the overall rejection areas (i.e., using the suprathreshold area statistic) to evaluate the overall significance of the experimental results $[9$.

After fixing the template parametrization, we used Log-Euclidean metrics to establish a metric on the surface deformation tensors at each point, and conducted a permutation test on the suprathreshold area of the resulting Hotellings $T^{2}$ statistics. The statistical map is shown in Figure 2(a). The threshold for significance at each surface point was chosen to be $p=0.05$. The permutation-based overall significance $p$ values, corrected for multiple comparisons, were $\mathrm{p}=0.0001$ for the right hemisphere and 0.0002 for the left hemisphere, respectively.

\subsection{Comparison with Other TBM Methods}

To explore whether our multivariate statistics provided extra power when running TBM on the surface data, in each experiment, we also conducted three additional statistical tests based on different tensor-based statistics derived from the Jacobian matrix. The other statistics we studied were: (1) the pair of eigenvalues of the Jacobian matrix, treated as a 2-dimensional vector; (2) the determinant of Jacobian matrix; and (3) the largest eigenvalue of Jacobian matrix. For statistics (2) and (3), we applied a Students $t$ test to compute the group mean difference at each surface point. In case (1), we used Hotelling's $T^{2}$ statistics to compute the group mean difference. For the three new statistics, their calculated statistical maps are shown in Figure 1(b)-(d), 1(f)-(h), and 2 (b)-(c), respectively. For each statistic, we also computed the overall $p$-values (see Table 1). In each experiment, the overall localization and spatial pattern of surface abnormalities detected by different tensor-based surface statistics were highly consistent. 


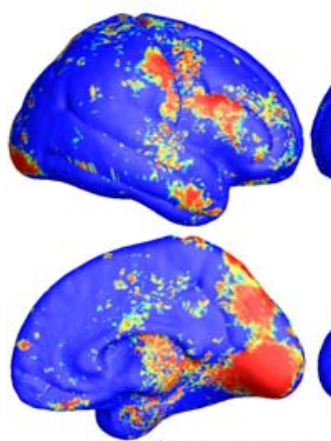

(a) Multivariate TBM

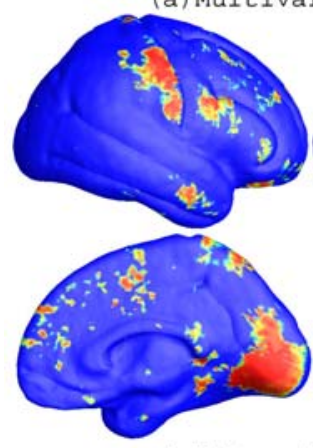

(c) Determinant of $\mathrm{J}$
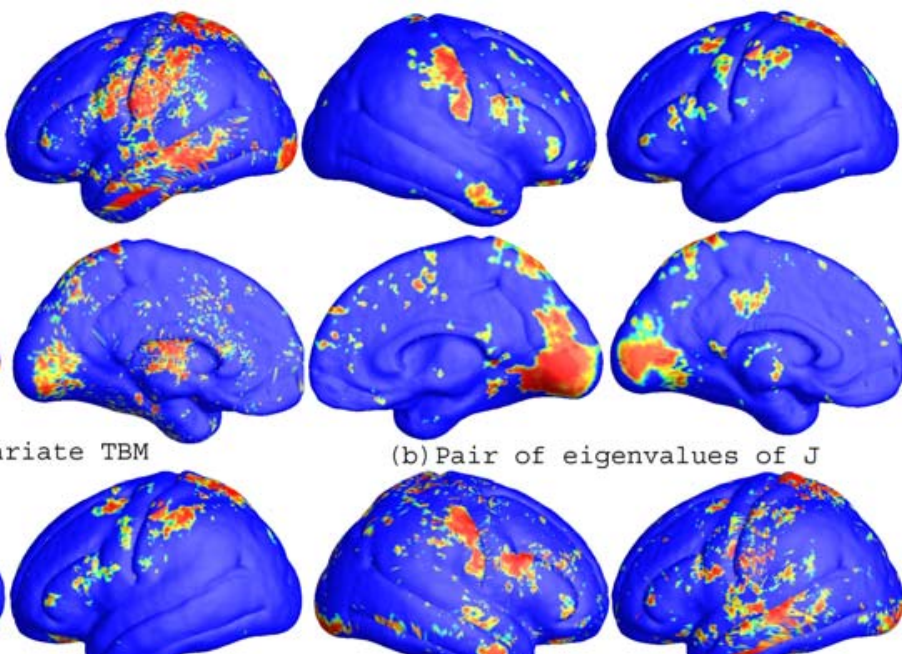

(b) Pair of eigenvalues of $\mathrm{J}$
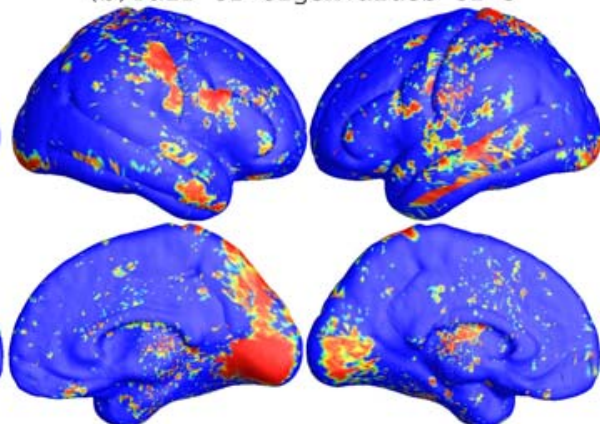

(d) The largest eigenvalue of $\mathrm{J}$

Fig. 2. (a)-(d) illustrate the slit map conformal parameterization on a right hemisphere cortical surface with 10 selected landmark curves. (e)-(h) are illustrative the statistical $p$-map results of multivariate TBM and other surface TBM for a cortical surface dataset from $40 \mathrm{WS}$ patients and 40 matched control subjects. The color-coded scale is the same as the one in Figure 1. where non-blue colors denote the vertices where there is a significant statistical difference, at the $p=0.05$ level. Multivariate statistics on the surface Jacobian matrix tend to detect group differences with the greatest effect sizes. Overall statistical significance values (corrected for multiple comparisons) are listed in Table 1

Table 1. Permutation-based overall significance $p$ value for three experiments. ( $J$ is the Jacobian matrix and EV stands for Eigenvalue. To detect group differences, it was advantageous to use the full tensor, or its two eigenvalues together; with simpler local measures based on surface area, group differences were missed.).

\begin{tabular}{|l|c|c|c|c|}
\hline & Full Matrix & Determinant of $J$ & Largest EV of $J$ & Pair of EV of $J$ \\
\hline Left Hippo Surface & $\mathbf{0 . 0 1 9 8}$ & 0.1446 & 0.1016 & 0.0474 \\
\hline Right Hippo Surface & $\mathbf{0 . 0 4 1 0}$ & 0.3600 & 0.3492 & 0.0688 \\
\hline Left Vent Surface & $\mathbf{0 . 0 0 2 8}$ & 0.0330 & 0.0098 & 0.0084 \\
\hline Right Vent Surface & $\mathbf{0 . 0 0 6 6}$ & 0.0448 & 0.0120 & 0.0226 \\
\hline Left Cortex & $\mathbf{0 . 0 0 0 2}$ & 0.1933 & 0.1627 & 0.0003 \\
\hline Right Cortex & $\mathbf{0 . 0 0 0 1}$ & 0.1366 & 0.1201 & 0.0002 \\
\hline
\end{tabular}


The experiments also strongly suggested that the newly proposed multivariate TBM method has more detection power in terms of effect size (and the area with suprathreshold statistics), probably because it captures more directional and rotational information when measuring geometric differences.

Acknowledgments. This work was funded by National Institute of Health through the NIH Roadmap for Medical Research, Grant U54 RR021813 entitled Center for Computational Biology (CCB).

\section{References}

1. Dale, A.M., Fischl, B., Sereno, M.I.: Cortical surface-based analysis I: segmentation and surface reconstruction. Neuroimage 9, 179-194 (1999)

2. Ashburner, J., Hutton, C., Frackowiak, R., Johnsrude, I., Price, C., Friston, K.: Identifying global anatomical differences: deformation-based morphometry. Human Brain Mapping 6(5-6), 348-357 (1998)

3. Chung, M., Dalton, K., Davidson, R.: Tensor-based cortical surface morphometry via weighted spherical harmonic representation. IEEE Trans. Med. Imag. 27(8), 1143-1151 (2008)

4. Guggenheimer, H.W.: Differential Geometry. Dover Publications (1977)

5. Wang, Y., Gu, X., Hayashi, K.M., Chan, T.F., Thompson, P.M., Yau, S.-T.: Brain surface parameterization using riemann surface structure. In: Duncan, J.S., Gerig, G. (eds.) MICCAI 2005. LNCS, vol. 3750, pp. 657-665. Springer, Heidelberg (2005)

6. Wang, Y., Gu, X., Chan, T.F., Thompson, P.M., Yau, S.T.: Conformal slit mapping and its applications to brain surface parameterization. In: Metaxas, D., Axel, L., Fichtinger, G., Székely, G. (eds.) MICCAI 2008, Part I. LNCS, vol. 5241, pp. 585593. Springer, Heidelberg (2008)

7. Leporé, N., Brun, C., Chou, Y.Y., Chiang, M.C., Dutton, R.A., Hayashi, K.M., Luders, E., Lopez, O.L., Aizenstein, H.J., Toga, A.W., Becker, J.T., Thompson, P.M.: Generalized tensor-based morphometry of HIV/AIDS using multivariate statistics on deformation tensors. IEEE Trans. Med. Imag. 27(1), 129-141 (2008)

8. Arsigny, V., Fillard, P., Pennec, X., Ayache, N.: Log-Euclidean metrics for fast and simple calculus on diffusion tensors. Magn. Reson. Med. 56, 411-421 (2006)

9. Thompson, P.M., Hayashi, K.M., de Zubicaray, G.I., Janke, A.L., Rose, S.E., Semple, J., Hong, M.S., Herman, D.H., Gravano, D., Doddrell, D.M., Toga, A.W.: Mapping hippocampal and ventricular change in Alzheimer's disease. NeuroImage 22(4), 1754-1766 (2004)

10. Thompson, P.M., Lee, A.D., Dutton, R.A., Geaga, J.A., Hayashi, K.M., Eckert, M.A., Bellugi, U., Galaburda, A.M., Korenberg, J.R., Mills, D.L., Toga, A.W., Reiss, A.L.: Abnormal cortical complexity and thickness profiles mapped in Williams syndrome. J. Neuroscience 25(16), 4146-4158 (2005)

11. Thompson, P.M., Dutton, R.A., Hayashi, K.M., Lu, A., Lee, S.E., Lee, J.Y., Lopez, O.L., Aizenstein, H.J., Toga, A.W., Becker, J.T.: 3D mapping of ventricular and corpus callosum abnormalities in HIV/AIDS. NeuroImage 31(1), 12-23 (2006)

12. Thompson, P.M., Woods, R.P., Mega, M.S., Toga, A.W.: Mathemati$\mathrm{cal} /$ computational challenges in creating population-based brain atlases. Human Brain Mapping 9(2), 81-92 (2000) 